\title{
Eine gemeinsame Aufgabe von Erwachsenen- sowie Kinder- und Jugendpsychiatrie
}

\author{
Die psychische Erkrankung eines Elternteils stellt für Kinder einen bedeutsamen \\ Risikofaktor in ihrer Entwicklung dar. Um ihre Situation zu verbessern, haben die \\ Psychiatrischen Dienste und die Kinder- und Jugendpsychiatrie des Kantons \\ Graubünden ein Konzept entwickelt, das die altersangemessene Psychoedukation \\ der Kinder und Unterstützung der Eltern in den Mittelpunkt stellt.
}

Elisabeth Schmidt ${ }^{a}$, Suzanne von Blumenthal , Jörg Leenersc

a Fachpsychologin für Psychotherapie, Kinder- und Jugendpsychiatrie Graubünden

b Dr. med., Chefärztin Psychiatrische Dienste Graubünden

c Dr. med., Chefarzt Kinderund Jugendpsychiatrie Graubünden
Korrespondenz:

Elisabeth Schmidt

Kinder- und Jugendpsychiatrie Graubünden KJP

Ambulante Angebote

Masanserstrasse 14

CH-7000 Chur

Tel. 0812529023

Fax 0812529501

elisabeth.schmidt[at]kjp-gr.ch
Kinder von psychisch kranken Eltern sind einem stark erhöhten Risiko ausgesetzt, im Lauf ihres Lebens selbst an einer psychischen Störung zu erkranken. Neben dem genetischen Risiko kann die psychische Erkrankung die Beziehungsfähigkeit und Erziehungskompetenz eines Elternteils beeinträchtigen. Des Weiteren sind die Kinder gehäuft psychosozialen Risiken ausgesetzt wie Armut, niedrigem sozioökonomischem Status der Eltern, Verlust von Bezugspersonen, Vernachlässigung, Misshandlung oder sexuellem Missbrauch [1, 2]. Mattejat und Remschmidt stellten fest, dass bei der Inanspruchnahmepopulation einer stationären kinder- und jugendpsychiatrischen Behandlung bis zur Hälfte der Kinder und Jugendlichen bei einem psychisch erkrankten Elternteil aufwuchsen [1].

Gemäss einer Nationalfonds-Studie aus dem Jahr 2006 haben in der Schweiz rund 50\% der psychisch Erkrankten Kinder. Schätzungen zufolge sind damit ca. 50000 Kinder betroffen, wobei man von einer hohen Dunkelziffer ausgehen muss [3]. Rechnet man allein die Kinder aus Familien mit Suchterkrankungen hinzu, erhöht sich die Zahl massiv. Nach Schätzungen der Schweizerischen Fachstelle für Alkohol- und andere Drogenprobleme (SFA) leben rund 100000 Kinder und Jugendliche in Familien mit einem suchtmittelabhängigen Elternteil [4].

Hilfe für die betroffenen Kinder besteht in erster Linie in der effektiven Behandlung der elterlichen Krankheit. Daneben sind weitere Bestandteile der Präventionsarbeit psychoeduktive Interventionen sowie therapeutische oder pädagogische Hilfen für Eltern und Kinder, die individuell an die Situation der Familie angepasst werden [1]. Studien haben gezeigt, dass ein hoher Informationsgrad der Kinder über die elterliche Erkrankung ein sehr bedeutender Schutzfaktor ist [5]. Die zentrale Frage in der Prävention ist daher, wie man die betroffenen Kinder erreichen kann, bevor sie selbst Auffälligkeiten entwickeln, bzw. sie bei Bedarf möglichst rasch in Kontakt mit spezialisierten Hilfsangeboten bringen kann.
Programme de prévention du canton des Grisons pour les enfants de parents souffrant de troubles psychiques: une tâche commune à la psychiatrie d'adultes et à la psychiatrie d'enfants et d'adolescents

Les troubles psychiques d'un des parents présentent pour les enfants un facteur de risque dans leur développement. Or, les enfants ne sont souvent pas pris en considération dans le traitement psychiatrique des parents. Pour améliorer la situation de ces enfants, les services de psychiatrie des Grisons (PDGR) et le service de psychiatrie pour enfants et adolescents des Grisons (KJP Graubünden) ont développé un concept qui met au centre la psychoéducation des enfants, adaptée à leur âge, et le soutien des parents. Après le succès de la phase pilote, le concept sera élargi à tous les services du canton où se trouvent des patients ayant des enfants mineurs.

\section{Projektaufbau und -ablauf}

Das gemeinsame Projekt der Psychiatrischen Dienste Graubünden (PDGR) und der Kinder- und Jugendpsychiatrie Graubünden (KJP Graubünden) wurde mit dem Ziel ins Leben gerufen, psychisch kranke Eltern für die Problematik zu sensibilisieren und sie dabei zu unterstützen, mit ihren Kindern altersgemäss über ihre Erkrankung zu reden. Auf diese Weise sollen Eltern und Kinder in der für alle Beteiligten schwierigen Situation entlastet und gestärkt werden. Darüber hinaus sollte die Möglichkeit einer weiterführenden Begleitung oder Abklärung in der KJP bei entsprechender Indikation vereinfacht werden. 
Die Erarbeitung des Konzepts begann im Oktober 2009. Die Projektgruppe setzte sich aus einem Pflegefachmann, einer Sozialarbeiterin und einem Psychiater der PDGR sowie einer Psychologin der KJP Graubünden zusammen. Von Oktober 2010 bis März 2011 fand eine sechsmonatige Pilotphase statt, in der das Konzept auf vier Stationen der Psychiatrischen Dienste überprüft wurde. Neben zwei Aufnahmestationen waren eine Mutter-Kind- sowie eine Station für Suchtpatienten beteiligt. Auf jeder Station waren zwei Pflegefachpersonen für die Umsetzung des Konzepts zuständig. Diese Verantwortlichen wurden zuvor in einer eintägigen Veranstal-

\section{Rund 100000 Kinder und Jugendliche leben in Familien mit einem suchtmittelabhängigen Elternteil.}

tung durch die KJP Graubünden in Bezug auf Entwicklungspsychologie, mögliche Reaktionsweisen von Kindern sowie Gesprächsführung mit Familien geschult und während der Pilotphase supervisorisch begleitet. Als Unterstützung in der Arbeit mit den Eltern und Kindern dienten Broschüren der pro juventute (z.B. «Wenn ein Vater oder eine Mutter psychische Probleme hat...») und von sucht info schweiz (z.B.«Kinder aus alkoholbelasteten Familien»). Auf Wunsch der Eltern bestand das Angebot gemeinsamer Gespräche mit den Kindern in der Erwachsenenpsychiatrischen Klinik mit Mitarbeitern der Kinder- und Jugendpsychiatrie. Für eine Überweisung zur KJP Graubünden galten folgende Kriterien: ausgeprägte Sorge der Eltern um die Entwick-

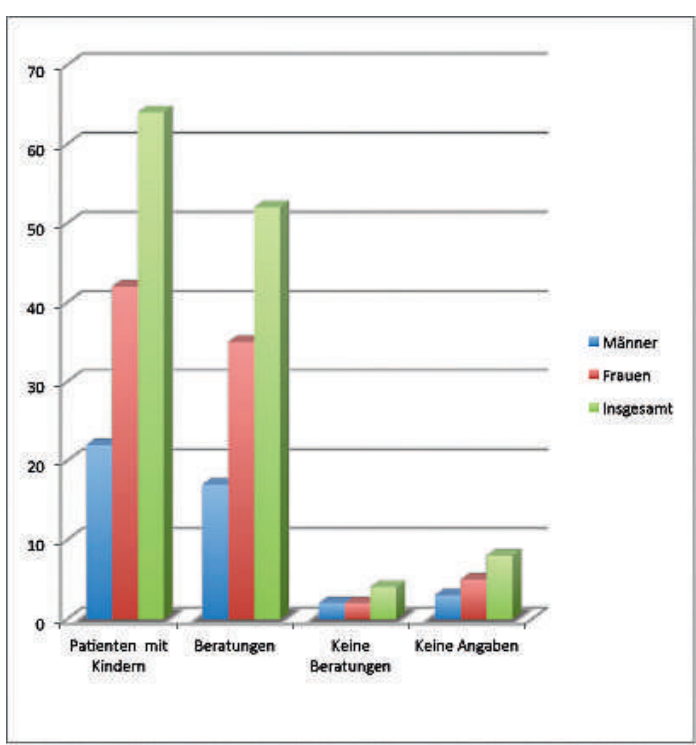

Abbildung 1

Patienten mit Kindern und Beratungsquote nach Geschlecht. lung, bereits vorliegende Schwierigkeiten der Kinder und Jugendlichen, Miterleben von potentiell traumatisierenden Ereignissen, wie z. B. Suizidversuch des Elternteils. Die Pflegefachpersonen hatten zudem immer die Möglichkeit, eine fragliche Indikation für eine Überweisung mit zuständigen Mitarbeitern der KJP Graubünden zu besprechen.

\section{Ergebnisse}

Während der sechsmonatigen Pilotphase wurden auf den vier Stationen insgesamt 212 Eintritte verzeichnet. Von den Patientinnen und Patienten hatten 30\% Kinder im unmündigen Alter; 42 der Frauen waren Mütter (35\%), 22 der Männer Väter (23\%) (Abb. 1). Alle Patientinnen und Patienten wurden nach der Situation ihrer Kinder gefragt, einerseits um sicherzustellen, dass diese in der Zeit des Klinikaufenthaltes gut betreut waren, andererseits um ihnen im Sinne des Projekts Unterstützung anzubieten, wie sie die Kinder angemessen über ihre Erkrankung informieren könnten. Während die Klärung der Betreuung am Beginn des Aufenthalts stand, wurde mit der Beratung gewartet, bis die Patientinnen und Patienten so weit stabil waren, um sich mit diesem Thema auseinandersetzen zu können. Teilweise kam ein verfrühter Austritt diesem Gespräch zuvor, oder die Patienten, häufig Väter, lebten schon seit Jahren getrennt von ihrer Familie und hatten keinen Kontakt zu den Kindern. Insgesamt war es in 81\% der Fälle möglich, Beratungen der Eltern durchzuführen (Abb. 2). In keinem der Fälle lag eine akute Gefährdung vor, in einem Fall wurde eine Notfallplazierung der Kinder während der stationären Behandlung in die Wege geleitet. In 5\% der Fälle fand eine kinderpsychiatrische Behandlung statt resp. wurden die Kinder zu einer Abklärung an die KJP überwiesen. Die Möglichkeit, eine Indikation zuvor mit der Kinder- und Jugendpsychologin des Projekts zu diskutieren, wurde von den Pflegefachpersonen sehr selten genutzt.

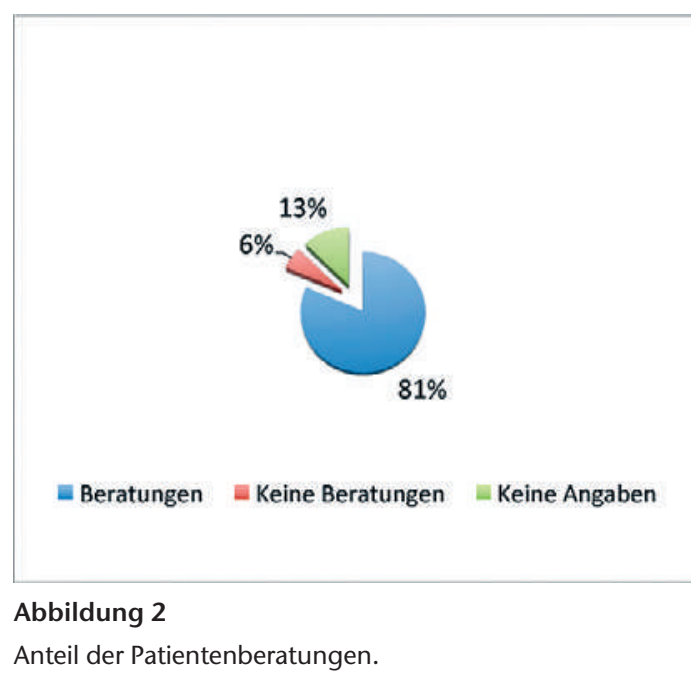




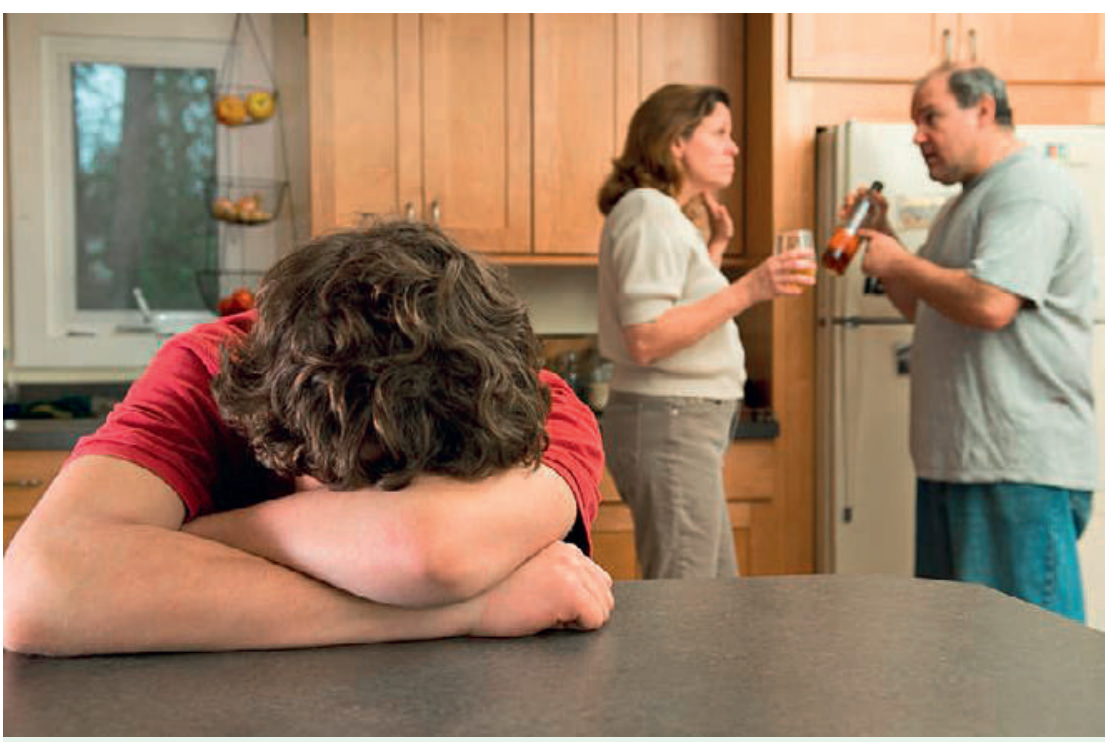

Hilfe für die Kinder von Suchtkranken besteht in erster Linie in der effektiven Behandlung der elterlichen Krankheit.

Bei Bedarf und Interesse wurden die Eltern mit Hilfe eines Flyers auf das Angebot der KJP hingewiesen, so dass sie sich auch zu einem späteren Zeitpunkt Unterstützung holen könnten. Bei den Patientinnen und Patienten wurde das Projekt weitgehend positiv aufgenommen. Deutliche Unterschiede zeigten sich jedoch zwischen den Stationen: Am einfachsten war die Umsetzung auf der Mutter-KindStation. Auf der Therapiestation der Suchterkrankungen war es hingegen deutlich schwieriger, einen förderlichen Austausch über die Situation der Kinder zu ermöglichen. Bis auf wenige Ausnahmen wurde von den Suchtpatientinnen und -patienten meist zurückgemeldet, dass es den Kindern gut gehe und kein Interesse an Unterstützung bestehe.

\section{Von den Betroffenen wird das Vorgehen}

\section{als hilfreich erlebt, von den Pflegefachpersonen als}

\section{Bereicherung beschrieben.}

Insgesamt wurde das Vorgehen von den Betroffenen positiv aufgenommen. So äusserten viele Eltern Erleichterung darüber, dass das Thema überhaupt angesprochen wurde und ihnen Handlungsmöglichkeiten aufgezeigt wurden, wie sie trotz ihrer Erkrankung ihre Elternrolle wahrnehmen können. Einige Patienten berichteten, dass sie selbst als Kinder von der Thematik betroffen waren und von einem solchen Angebot profitiert hätten. Auch aufseiten der Pflegefachpersonen stiess das Projekt auf Akzeptanz und Interesse, die zusätzlichen Aufgaben waren vom Umfang her gut neben dem Kernge- schäft umsetzbar. Aufgrund der guten Erfahrungen in der Pilotphase und der Wichtigkeit, die Situation der Kinder psychisch kranker Eltern zu verbessern, wird das Konzept in Zukunft auch auf fast alle anderen Stationen ausgeweitet. Ergänzend wurde seit Februar 2011 auf mehreren Stationen mit Elterngruppen begonnen, die Psychoedukation über die Situation der Kinder und die Möglichkeit zum Austausch der Eltern untereinander anbieten. Ziel der Elterngruppe ist es, den Eltern alltagsnah Kompetenzen im Umgang mit ihren Kindern und ihrem Partner zu vermitteln und diese anhand konkreter Beispiele gemeinsam einzuüben. Die Gruppe findet wöchentlich statt und wird interdisziplinär geleitet von ärztlich-psychologischer und pflegerischer Seite.

\section{Fazit}

Die Erfahrungen der Pilotphase haben gezeigt, dass es möglich ist, das Präventionsprogramm für Kinder psychisch kranker Eltern im Kontext der stationären Erwachsenenpsychiatrie mit verhältnismässig wenig Aufwand umzusetzen. Somit wird ein wichtiger gesellschaftlicher Beitrag geleistet, um psychisch kranke Eltern und ihre Kinder zu stärken und Fehlentwicklungen vorzubeugen. Von den Betroffenen wird das Vorgehen als hilfreich erlebt, von den Pflegefachpersonen als Bereicherung beschrieben.

Als einer der Erfolgsfaktoren hat sich die Implementierung des Konzepts mittels einer Schulung und begleitender Supervision erwiesen. Die Sensibilisierung der Pflegefachpersonen für die Situation der betroffenen Familien stellt unseres Erachtens eine zentrale Voraussetzung für eine Haltung dar, die die Patienten als Eltern wahrnimmt und stärken kann, jenseits ihrer Patientenrolle. Klare Zusammenarbeitsstrukturen zwischen PDGR und KJP Graubünden mit niederschwelligen Austauschmöglichkeiten haben ebenfalls zu einer effizienten Umsetzung beigetragen. Weiterhin hat sich zudem das Informationsmaterial von pro juventute und sucht info schweiz als hilfreich erwiesen.

Zu Beginn der Pilotphase äusserten sich mehrere Pflegefachpersonen skeptisch, ob sie der Zusatzaufgabe hinsichtlich ihrer zeitlichen und fachlichen Ressourcen gerecht werden könnten. Eine klare Abgrenzung der Zuständigkeiten, konkretes Einüben der Gespräche und das Beratungs- und Supervisionsangebot trugen dazu bei, dass sich die Pflegefachpersonen zunehmend sicherer und der Aufgabe gewachsen fühlten. Die positiven Erfahrungen und Rückmeldungen der Patienten bewirkten zudem eine Stärkung und kontinuierliche Kompetenzerweiterung, sodass die Pflegefachpersonen wiederum die Eltern wirksamer in ihrer Elternrolle unterstützen konnten. Allerdings hat sich trotz des relativ geringen zeitlichen Aufwands gezeigt, dass die Umsetzung des Konzepts durch eine hohe Arbeitsbelastung eingeschränkt wird. Es erscheint daher wichtig, mittels regelmässiger Supervisionen das Bewusstsein für 
die Problematik wiederholt zu thematisieren und zu vertiefen.

Im Vergleich zu den Zahlen der NationalfondsStudie [3] fanden sich in der Pilotphase verhältnismässig wenige Eltern unter den Patienten (30\%). Dies könnte daran liegen, dass nur Patienten mit Kindern in unmündigem Alter erfasst wurden. Die Grenzen des Konzepts zeigen sich in der Beratungsrate von $81 \%$. Das Angebot beruht auf Freiwilligkeit. Wenn Patientinnen und Patienten eine Beratung oder einen Einbezug der Kinder ablehnen, sei es weil sie ihre Erkrankung oder die Folgen für die Kinder

\section{Im Bereich Sucht scheint es besonders schwierig, den Teufelskreis aus Scham, Schuld, mangelnder Krank- heitseinsicht, Bagatellisierung und Tabuisierung zu durchbrechen.}

lichkeit, mit wenig Aufwand die Situation der betroffenen Familien verbessern zu können und Folgekosten zu vermindern, spricht eindeutig dafür, ein solches Vorgehen als Standard in der Schweiz einzuführen.

\section{Literatur}

1 Mattejat F, Remschmidt H. Übersichtsartikel Kinder psychisch kranker Eltern. Deutsches Ärzteblatt. 2008;105(23): 413-8.

2 Mattejat F, Lisofsky B. (Hrsg.). Nicht von schlechten Eltern: Kinder psychisch Kranker. Bonn: Balance buch + medien Verlag; 2008.

3 Sollberger D. Biographische Identität zwischen Stigma und Tabu. Kinder psychisch kranker Eltern Resultate einer Schweizerischen Nationalfonds-Studie. Der Nervenarzt 2007; 78 Suppl. 2: 491.

4 Stiftung Bündner Suchthilfe. Suchtreport der suchthilfe.gr. Jahresbericht 2011.

5 Lenz A. Interventionen bei Kindern psychisch kranker Eltern. Göttingen: Hogrefe Verlag; 2008.

bagatellisieren oder tabuisieren, ist dies zu respektieren. Im Bereich Sucht scheint es besonders schwierig, den Teufelskreis aus Scham, Schuld oder mangelnder Krankheitseinsicht, Bagatellisierung und Tabuisierung zu durchbrechen und mit Eltern die Situation ihrer Kinder zu thematisieren. Dennoch bleibt zu hoffen, dass diese Patientinnen und Patienten längerfristig sensibilisiert werden und sich die Wahrscheinlichkeit erhöht, die Bedürfnisse ihrer Kinder wahrzunehmen und spezialisierte Hilfsangebote in Anspruch zu nehmen.

Eine weitere Grenze des Konzepts besteht darin, dass ambulante Patientinnen und Patienten zurzeit noch nicht berücksichtigt werden können. Dafür wäre die Vernetzung und Zusammenarbeit mit niedergelassenen Psychiatern massgebend. Die Mög-

\section{Verwendete Broschüren}

- Wenn ein Vater oder eine Mutter psychische Probleme hat ... Informationen für Eltern. pro juventute (2007, 4. Aufl.)

- Wenn dein Vater oder deine Mutter in psychiatrische Behandlung muss ... Informationen für Kinder im Alter von 8-12 Jahren. pro juventute (2007, 4. Aufl.).

- Wenn dein Vater oder deine Mutter psychische Probleme hat ... Informationen für Jugendliche zwischen 12 und 18 Jahren. pro juventute (2007, 4. Aufl.).

- Kinder aus alkoholbelasteten Familien. Schweizerische Fachstelle für Alkohol- und andere Drogenprobleme (2004).

- Eltern vor allem - Eltern trotz allem. Sucht Info Schweiz (2010). 